\title{
Research on Solids Conveying Capacity of Gas Solids Pneumatic Conveying Fly Ash in Up-extraction Fluidized Bin System
}

\author{
DUAN Guangbin ${ }^{a}$, WANG Wenzhi ${ }^{b}$, LIU Feng ${ }^{c}$, LI Jinkaid and LIU Zongming ${ }^{\mathrm{e}}$ * \\ School of Materials Science and Engineering, University of Jinan, Shandong, China \\ amse_duangb@ujn.edu.cn, ${ }^{b} \mathrm{mse}$ _wangwz@163.com, ${ }^{\mathrm{c}} \mathrm{mse}$ liuf@163.com, ${ }^{\mathrm{d}} \mathrm{mse}$ _lijk@ujn.edu.cn, \\ est_liuzm@ujn.edu.cn,
}

Keywords: positive pneumatic conveying, conveying capacity, constant characteristics, Up-extraction

Abstract. This paper reported the characteristics of solids conveying capacity of gas solids pneumatic conveying fly ash in up-extraction fluidized bin system. Up-extraction pneumatic conveying experiment bench was formed based on industrial case. Meanwhile automatic control experimental system which included fluidized gas control parameter, conveying gas control parameter, compressed gas control parameter, and solids mass flux ratio etc was built up. Fly ash particles were conveyed in this experimental system. As a result, the trends of system conveying capacity in different transmission conditions were given. The methods of adjusting solids conveying capacity were obtained too by the changing of fluidized gas control value, conveying gas control value and compressed gas control parameters.

\section{Introduction}

As an prevalent solids conveying style, pneumatic conveying played an important role in the industrial process, which was widely used in power engineering, food industry, building materials [1], chemical industry, metallurgy and other fields because of its merits such as energy consumption, low wear, flexible installation and automatic control [2]. Nowadays, the conveying energy consumption and stability was mentioned and solids flux ratio was also obtained in process of pneumatic conveying [3].

Conveying bin was the critical equipment in positive pressure pneumatic conveying system. But the related research of bin was less except numerical simulation the conveying process. Of course, the solids quantitative conveying report was so lack that we must carried out this issue. In this case, Chen Juhui simulated gas solid flow characteristics in fluidized bin based on experimental data. Kawaguchi and Tsuji obtained turbulence properties of particle force behavior in down-push conveying bin according to discrete hard-sphere model. Omita researched solid flow ratio of up-discharge and gave the solid flow ratio calculated equation, meanwhile the feeder bin size influenced solids conveying properties less. Guo Xiaolei designed lucite feeder bin and gave experiments results of transporting coal fines. Jones believed that the distance between pipe and fluid-bed influenced conveying parameters obviously [4].

In engineering application, pneumatic conveying system occurred to feed solids non-homogeneously which constrained the development of this type deliver style. The up-extraction fluidized bin was widely used because of simple structure and smooth flow. But the suspension effectiveness and concentration in feeder was inhomogeneous and difficult to control solid discharge. It affected the application effect of bin feeder. Up-extraction fluidized bin system was used widely for powder with good fluidization distinction, which Fly ash was included.

In this paper, up-extraction fluidized bin pneumatic conveying system was designed and fabricated. Gas solid flow experiments were carried out by conveying fly ash particles. Based on the experimental data, the trend of system conveying capacity, vs gas velocity, pneumatic pressure etc flow parameters was performed in detail. Meanwhile, auto control system was built up according to experiment process. By adjustment and control, solid flow ratio could be adjusted which set solid capacity equilibriums. 


\section{Experimental SET-UP}

In this paper, the conveying tests were performed in a $0.08 \mathrm{~m}$ I.D pipe flow conveying system. The transporting system (see Fig.1) was made up of gas supply, feeder, collecting system, data acquisition system, and pipeline.

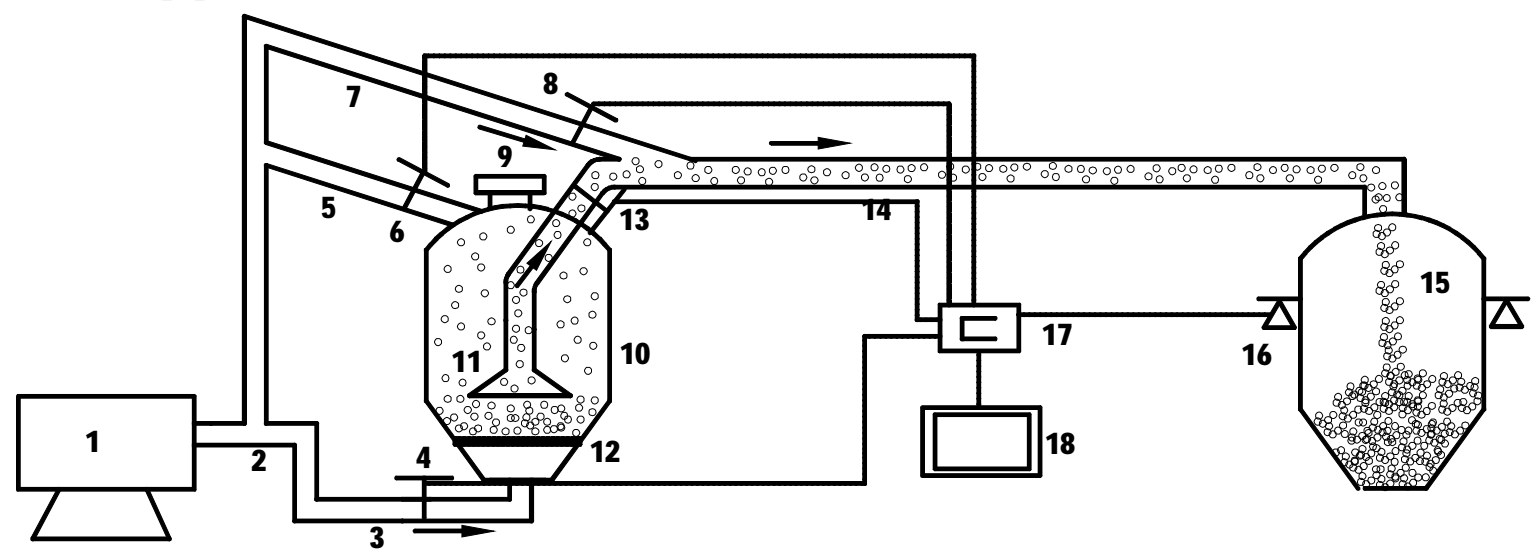

Fig.1. Diagram of experimental system

1. gas compressor 2. gas pipeline 3. fluidization gas pipeline 4. fluidization gas control valve 5 . pusher pipeline 6. pusher pipeline control valve 7 . conveying gas pipeline 8 . conveying gas control valve 9 . feed inlet 10 . feeder bin 11 . discharge 12. fluidized bed 13. conveying pipeline control valve 14. conveying pipeline 15 . receiving bin 16 . weighing sensor 17 . data acquisition card 18. computer

In this paper, fly ash was fed in conveying bin and then the feed inlet was closed by butterfly valve. The gas compressor was opened and compressed air entered the feeder bin. Fly ash were fluidized and then the static pressure arrived a constant value. The gas solid two phase flow entered pipe, and finally reached the receiving bin. In this experiment, the solid particles concentration decreased gradually until no particles in pipe. In process of conveying solids, trend of solid flow ratio by monitoring the change of high accuracy weighing sensors was given. Meanwhile the paper established PID control system. Of course, we can adjust solid flow ratio by setting the opening controller valve of fluidization gas, pusher gas, conveying gas, which can achieve solid flow constantly.

$360 \mathrm{~kg}$ fly ash was placed in feeder bin, which was about $2 / 3$ of feeder volume. The material was dry fly ash and its physical parameters were shown in Table 1.

Table 1 Material properties

\begin{tabular}{ccccc}
\hline Powder & $\begin{array}{c}\text { Equivalent spherical } \\
\text { diameter } \\
{[\mu \mathrm{m}]}\end{array}$ & $\begin{array}{c}\text { Particle density } \\
{\left[\mathrm{kg} / \mathrm{m}^{3}\right]}\end{array}$ & $\begin{array}{c}\text { Bulk density } \\
{\left[\mathrm{kg} / \mathrm{m}^{3}\right]}\end{array}$ & Sphericity \\
\hline Fly ash & 34.03 & 2038 & 771.5 & 0.95 \\
\hline
\end{tabular}

\section{Experimental Results}

Trend of solid conveying capacity in different time interval in case of opening fluidization gas pipeline. Fig. 2 showed the curve of solids conveying capacity vs time, as the fluidization gas and conveying pipeline control valves opening. The gas velocity was $9.85 \mathrm{~m} / \mathrm{s}$ and the conveying pressure was set $0.30 \mathrm{MPa}$. 


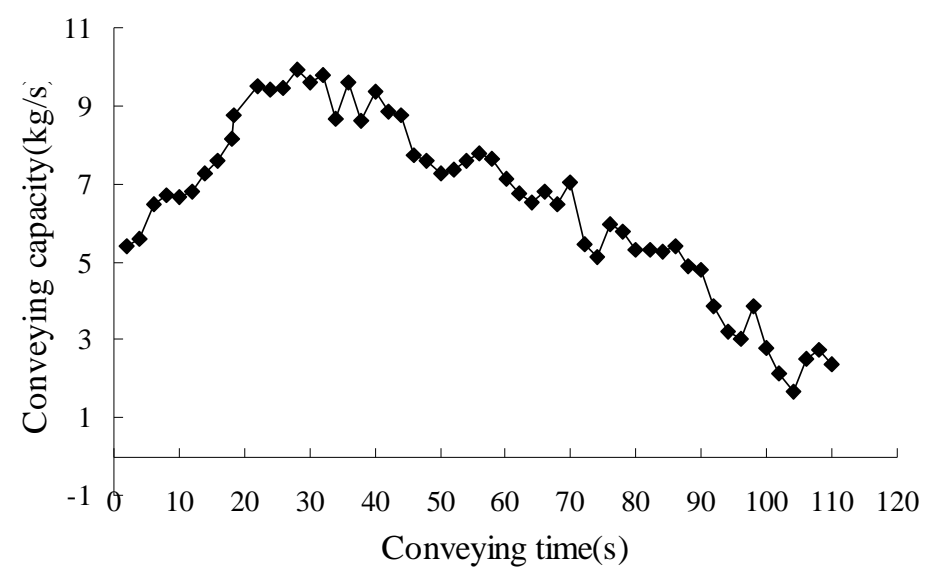

Fig.2. Solids conveying capacity vs conveying time when open fluidization gas pipeline

From this figure, the solids conveying capacity increased as time flow, if the superficial velocity and pressure of gas had been fixed. The reason was that when the time increased, there would be more particles in unit volume. Thus force of particles or between particles and the pipe wall would be added, and the solids conveying capacity increased. The conveying kept constant value for a moment and then decreased immediately. The stable conveying sustained less, and the rangeability of solid flow ratio was much larger. It was because that, as conveying continued, the solid particles mass decreased, which led to the solid flux reducing down. Simultaneously, the conveying capacity fluctuated greatly because of unstable solid flux. According to the experimental data, the conveying capacity of stable course error ranged in $\pm 17 \%$.

Trend of solid conveying capacity in different time interval in case of opening fluidization gas pipeline and conveying pipeline.

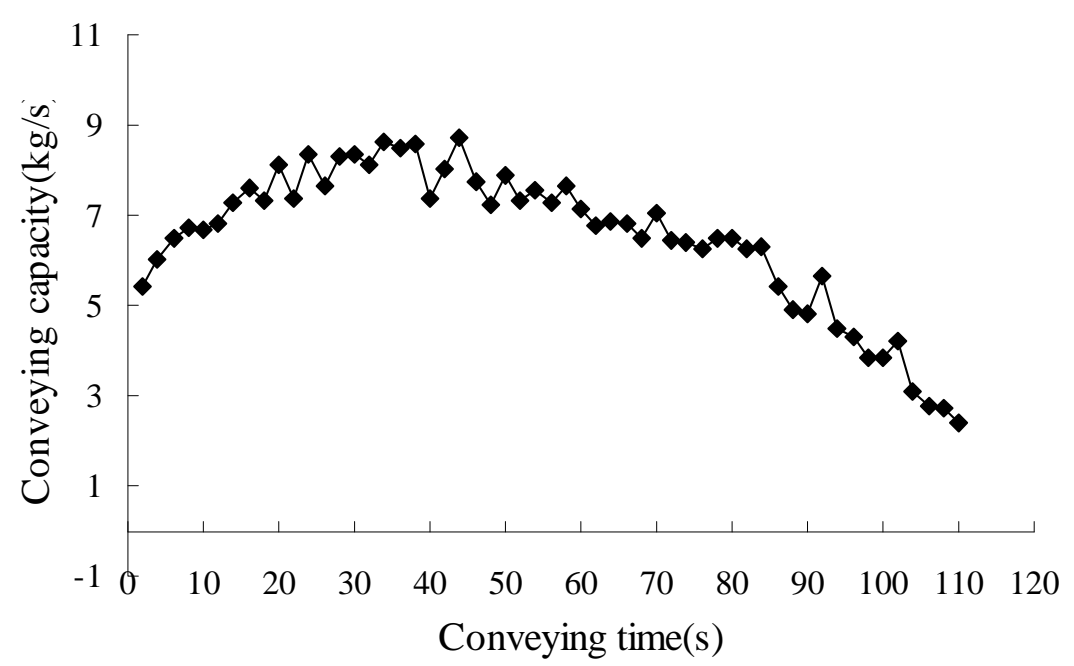

Fig.3. solids conveying capacity vs conveying time when open fluidization gas pipeline and conveying pipeline

Fig.3 showed the curve of solids conveying capacity vs time, as the fluidization gas, conveying gas pipeline and conveying pipeline control valves opening. The gas velocity was $9.85 \mathrm{~m} / \mathrm{s}$ and the conveying pressure was $0.30 \mathrm{MPa}$ too. From this figure, compared to the results above, the solids conveying capacity increased as time flow, meanwhile the conveying capacity kept constant value for a moment and then decreased immediately. But the stable process of gas solid flow was much longer. Solids flow kept smooth. The reason for this phenomenon was gas pressure and flow in pipeline increased because of opening conveying gas pipeline which led to solid concentration stable. The addition of gas came from conveying pipeline weakened the pulsation of gas in conveying pipe. According to the experimental data, the conveying capacity of stable course error ranged in $\pm 10 \%$. 
Trend of solid conveying capacity in different time interval in case of opening fluidization gas pipeline and pusher gas pipeline.

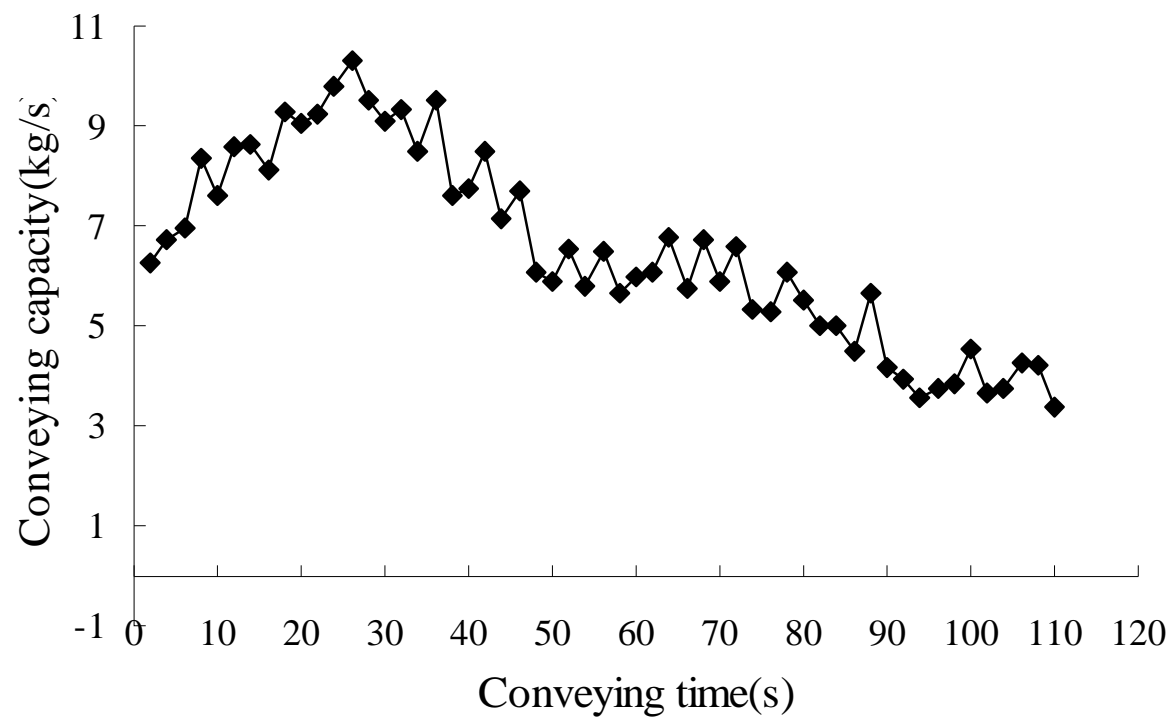

Fig.4. Solids conveying capacity vs conveying time when open fluidization gas pipeline and pusher gas pipe

Fig.4 gave the curve of solids conveying capacity vs time, as the fluidization gas and pusher gas pipeline control valves opening. The gas velocity was $9.85 \mathrm{~m} / \mathrm{s}$ and the conveying pressure was 0.30MPa too. From this figure, compared to the results of Fig.3 and Fig.4, the solids conveying capacity increased as time flow, and then kept constant value for a moment then decreased. But the stable process of gas solid flow was less duration. The reason for this phenomenon was pushed gas pipeline supplied the loss of fluidization gas pressure and flow ratio which led to stable state in feeder bin. But meanwhile, gas flow ratio and solid capacity in conveying pipe increased which meant the impetus of gas solid two phase and pulsation effect extended. According to the experimental data, the conveying capacity of stable course error ranged in $\pm 14 \%$. By comparing Fig.3 and Fig.4, the adjustment effect of modulation for fluidization gas pipeline and conveying pipe was much valid.

Trend of solid conveying capacity in different time interval in case of opening fluidization gas pipeline, conveying gas pipeline and pusher gas pipeline.

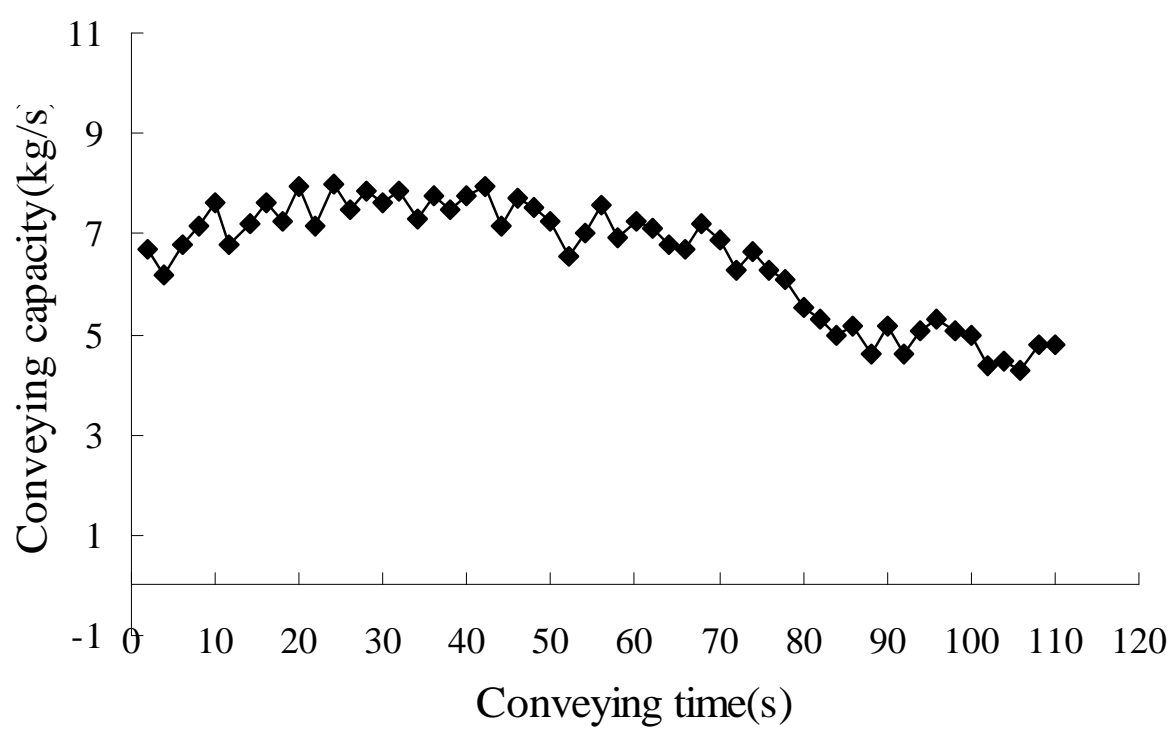

Fig.5 Solids conveying capacity vs conveying time when open fluidization gas pipeline, conveying gas pipeline and pusher gas pipe

Fig.5 gave the curve of solids conveying capacity vs time, as the fluidization gas, conveying gas pipeline and pusher gas pipeline control valves opening. The gas velocity was $9.85 \mathrm{~m} / \mathrm{s}$ and the 
conveying pressure was $0.30 \mathrm{MPa}$ too. From this figure, compared to the results of Fig.2, Fig.3 and Fig.4, the solids conveying capacity kept constant value for long time. Although the solid flow ratio decreased with reduction of solids level in feeder bin, we can keep stable by timely adjustment of gas valves. By comparing Fig.3, Fig.4 and Fig.5 the adjustment effect of modulation was most valid and its maximum error was less than $7 \%$.

\section{Conclusions}

In this paper, up-extraction pneumatic conveying experiment bench was formed according to industrial case. Automatic control experimental system which included fluidized gas control parameter, conveying gas control parameter, compressed gas control parameter, and solids mass flux ratio etc was achieved. The trend of solid conveying capacity by adjusting fluidization gas pipeline, conveying gas pipeline and pusher gas pipeline was given by transmitting fly ash.

According to experimental research, the solids conveying capacity increased to a constant value and kept for a moment, but decreased finally. Meanwhile we concluded that the conveying stable effect of adjusting fluidization gas, conveying gas and pusher gas was much effective than that of adjustment for other conditions.

\section{Acknowledgements}

This work was supported by Shandong Provincial Technology Development Project(Grant No.2013GGX10308), Natural Science Foundation of Shandong Province(Grant No.ZR2015EL037), Shan dong Provincial Technology Development Project(Grant No.2014GGX105007), Jinan Technology Development Project(Grant No. 201202071)

\section{References}

[1] C. Wagner, F.N. Ihunegbo, M. Halstensen and K.H. Esbensen: Powder Technol. Vol. 237 (2013), p. $506-513$

[2] S.S. Mallick and P.W. Wypych: Powder Technol. Vol. 194 (2009), p. 181-186

[3] R. Evgeny and K. Haim: Powder Technol. Vol. 207 (2011), p. 119-133

[4] C. Liang, L. Shen, P. Xu, G. Xu, G. Yuan, X. Chen and Zhao C: Exp. Therm. Fluid. Sci. Vol. 57 (2014), p. 11-19 